Andrea E. Delitto, $\mathrm{PhD}^{1^{*}}$, Fernanda Rocha, DMD, $\mathrm{PhD}^{1^{*}}$, Ann M. Decker, $\mathrm{DMD}^{2}$, Byron Amador ${ }^{1}$, Heather L. Sorenson ${ }^{1}$, and Shannon M. Wallet, $\mathrm{PhD}^{1 \wedge}$.

${ }^{1}$ Department of Oral Biology, University of Florida, Gainesville, FL 32610, ${ }^{2}$ Department of Periodontology, University of Michigan.

${ }^{*}$ contributed equally to the publication.

^Corresponding Author:

Shannon M. Wallet, PhD

Associate Professor

College of Dentistry

Department of Oral Biology

PO BOX 100434

Gainesville, FI 32610

$352-273-8370$

swallet@dental.ufl.edu

abstract word count (300): 294

total word count (3200): 3200

number of tables/figures (5): 3

number of references (40): 26

key words: epithelial cells, MyD88, periodontal disease, inflammation, homeostasis 


\section{ABSTRACT (294)}

Periodontal diseases are a class of non-resolving inflammatory diseases, initiated by a pathogenic subgingival biofilm, in a susceptible host, which if left untreated can result in soft and hard tissue destruction. Oral epithelial cells are the first line of defense against microbial infection within the oral cavity, whereby they can sense the environment through innate immune receptors including toll-like receptors (TLRs). Therefore, oral epithelial cells directly and indirectly contribute to mucosal homeostasis and inflammation, and disruption of this homeostasis or over-activation of innate immunity can result in initiation and/or exacerbation of localized inflammation as observed in periodontal diseases. Dynamics of TLR signaling outcomes are attributable to several factors including the cell type on which it engaged. Indeed, our previously published data indicates that oral epithelial cells respond in a unique manner when compared to canonical immune cells stimulated in a similar fashion. Thus, the objective of this study was to evaluate the role of oral epithelial cell innate sensing on periodontal disease, using a murine poly-microbial model in an epithelial cell specific knockout of the key TLRsignaling molecule MyD88 (B6 $\left.{ }^{\mathrm{K} 5 \mathrm{Cre} . M y D 88 p l o x}\right)$. Following knockdown of MyD88 in the oral epithelium, mice were infected with Porphorymonas gingivalis and Aggregatibacter actinomycetemcomitans by oral lavage 4 times per week, every other week for 6 weeks. Loss of oral epithelial cell MyD88 expression resulted in exacerbated bone loss, soft tissue morphological changes, soft tissue infiltration, and soft tissue inflammation following polymicrobial oral infection. Most interestingly while less robust, loss of oral epithelial cell MyD88 also resulted in mild but statistically significant soft tissue inflammation and bone loss even in the absence of a polymicrobial infection. Together these data demonstrate that oral epithelial cell MyD88-dependent TLR signaling regulates the immunological balance within the oral cavity under conditions of health and disease. 


\section{INTRODUCTION}

Periodontal diseases are a class of inflammatory diseases that if left untreated can result in soft and hard tissue destruction surrounding the tooth (Blinkhorn et al., 2009). While microbes are etiological agents of periodontal disease, they do not directly cause disease, but rather induce harmful inflammatory responses in a susceptible host(Page \& Kornman, 1997). Specifically, in periodontal disease, subgingival bacteria initiate and sustain a non-resolving inflammation that is ineffective at controlling the infection (Gaffen \& Hajishengallis, 2008).

Oral epithelial cells are the first line of defense against microbial infection within the oral cavity. These important cells serve multiple roles including: 1. a physical barrier to microbes; 2. a source of anti-microbial peptides which inhibit microbe growth; 3. innate immune cells which produce cytokines to trigger anti-microbial immunity; and 4. tolerogenic immune cells which can produce immunoregulatory cytokines or remain quiescent in response to non-pathogenic commensal microorganisms (McCormick \& Weinberg). We and others have demonstrated that the oral epithelial cell express several innate immune receptors including toll-like receptors (TLRs), whereby their ligation induces immune and anti-microbial factors (Dale et al., 2001; Diamond, Kimball, Krisanaprakornkit, Ganz, \& Dale, 2001; Ford, Gamonal, \& Seymour, 2010; Kollisch et al., 2005; Mahanonda \& Pichyangkul, 2007; Sugawara et al., 2006; Weinberg, Krisanaprakornkit, \& Dale, 1998). To this end, our previously published data indicates that oral epithelial cells respond in a unique manner when compared to canonical immune cells stimulated in a similar fashion (Wallet et al., 2013), but the relative role of this innate immune function to the protection from or induction of periodontal disease was not evaluated.

TLRs have two distinct functions on epithelial cells - protection from infection (immune activation) and tissue homeostasis (immune regulation) (Shibolet \& Podolsky, 2007). Therefore, over-activation of innate immunity or disruption of this homeostasis can result in initiation and/or exacerbation of localized inflammation as seen in periodontal diseases. TLRs utilize both MYD88-depedent and MYD88-independent signaling pathways which, at least in macrophages, are responsible for induction of pro-inflammatory cytokines or type 1 interferon and interferon-inducible genes, respectively (Lu, Yeh, \& Ohashi, 2008). Here we tested the contribution of the MyD88-dependent innate sensing of oral epithelial cells to periodontal disease using a novel cell-specific knockdown. Inducible knockout of MyD88 in oral epithelial cells exacerbated bone loss and soft tissue inflammation following a polymicrobial oral infection. Additional data indicates that MyD88-dependent 
signaling in oral epithelial cells also plays a role in controlling inflammation even in the absence of infection. Together these data suggest that innate immune sensing by oral epithelial cells is key to controlling inflammation under conditions of health and disease.

\section{MATERIALS AND METHODS}

Murine Models. All experimental procedures were conducted in accordance with the guidelines of the University of Florida Institutional Animal Care and Use Committee. All mice were maintained in a specific pathogen-free (SPF) environment at the breeding facilities of the University of Florida. C57BI/6 (B6) Keratin CrePR mice $\left(\mathrm{B}^{\mathrm{K} 5 \mathrm{Cre}}\right)$ were a kind gift from Dr. Xiao-Jing Wang from the University of Colorado Denver Health Sciences Center (Malkoski, Cleaver, Lu, Lighthall, \& Wang, 2010). Keratin CrePR mice express a Cre recombinase progesterone receptor (PR) fusion protein whose expression is restricted by a Keratin 5 (K5) promoter. The CrePR is inducible by a progesterone antagonist but not by endogenous progesterone (Malkoski et al., 2010). Upon induction with RU486, CrePR translocates into the nucleus and excises DNA

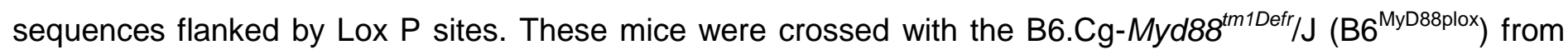
Jackson Laboratories (Bar Harbor, ME), which bare loxP sites on either side of exon 3 of the MyD88 gene. When these mutant mice are bred to $\mathrm{B}^{\mathrm{K} 5 \mathrm{Cre}}$ mice, offspring ( $\left.\mathrm{B} 6^{\mathrm{K} 5 \mathrm{Cre} . \mathrm{MyD} D 8 \mathrm{pplox}}\right)$ will have MyD88 deleted in cells with an active $\mathrm{K} 5$ promotor upon the administration of a progesterone antagonist.

Induction of Periodontal Disease. MyD88 in the oral epithelial cells was either knocked down or not through the oral administration of 500ug of RU486 (Sigma Aldrich, St. Louis, MO) solubilized in 25uL of sesame oil (Publix, Gainesville, FL) administered one time a day for three consecutive days. After which all mice were lavaged with $25 \mathrm{ul}$ of $0.12 \%$ chlorhexidine gluconate (3M, St. Paul, MN) for three days. Infection consisted of an 25ul oral lavage with $2.5 \times 10^{9}$ Porphoromonas gingivalis strain 381 and $2.5 \times 10^{9}$ Aggregatibacter actinomycetemocomitans strain 29522 (ATCC, Manassas, VA) resuspended in 2\% low viscosity carboxymethyl-cellulose (Sigma-Aldrich) on 4 consecutive days, every other week for 6 weeks. Each week of infection, on the first day of infection, prior to infection, microbial sampling of the oral environment was performed with calcium alginate swabs (Fisher Scientific). One week following the last infection, the maxillae and mandibles were harvested to evaluate bone morphometric analysis, soft tissue infiltration, and/or soluble mediator expression. 
Bacterial Growth. Both bacterial strains were grown under anaerobic conditions $\left(85 \% \mathrm{~N}_{2}, 10 \% \mathrm{H}_{2}, 5 \% \mathrm{CO}_{2}\right)$ at $37^{\circ} \mathrm{C}$ in a Coy anaerobic chamber. All media were fully reduced for 24 to $48 \mathrm{~h}$ prior to inoculation. $P$. gingivalis strain 381 was grown for 3 days on anaerobic 5\% sheep blood agar plates (Remel, Lenexa, KS), and the resultant growth scraped using a sterile inoculating loop and placed in trypticase soy broth supplemented with $0.5 \%$ yeast extract, $0.05 \% \mathrm{~L}$-cysteine, hemin $\left(5 \mu \mathrm{g} \mathrm{ml}^{-1}\right)$, and vitamin $\mathrm{K}_{1}\left(1 \mu \mathrm{g} \mathrm{ml}^{-1}\right)$ and allowed to culture anaerobically at $37^{\circ} \mathrm{C}$ until reaching an $\mathrm{OD}$ of 0.53 . A. actinomycetemocomitans strain 29522 was grown in Trypticase Soy Broth supplemented with 0.5\% yeast extract (TSB-YE) in a humidified, $10 \% \mathrm{CO}_{2}$ atmosphere, at $37^{\circ} \mathrm{C}$ until reaching an $\mathrm{OD}$ of 0.70 .

Alveolar Bone Loss. The maxilla of mice were de-fleshed and immersed in 3\% hydrogen peroxide (Fisher Scientific). To quantify bone loss, color digital images were captured under a 10x stereo dissecting microscope (Leica, Buffalo Grove, IL). Using morphometry image analysis software, the area of lingual bone resorption in $\mathrm{mm}^{2}$ was determined. The volumes of interests (VOI) were identified and an appropriate and uniform threshold was applied to all specimens after comparing grayscale and binarized images in all groups. After thresholding, the bone volume/bone volume of WT was quantified.

Periodontal Tissue Inflammatory Scoring. The left maxilla of all mice were removed and fixed in $10 \%$ buffered formalin, decalcified, embedded and sectioned for histological analysis. 5- $\mu \mathrm{m}$ sections were deparafinzed in xylene (25min), followed by stepwise rehydration in 100\% (5min) 95\% (5min), and $70 \%$ (5min) ethanol. Rehydrated sections were then rinsed in distilled water ( $5 \mathrm{~min})$ followed by equilibration in PBS ( $5 \mathrm{~min})$. Sections were stained with hematoxylin and eosin (Sigma-Aldrich). Images were captured at 20x magnification and inflammation scored using $\mathrm{PMN} /$ mononuclear cell infiltration ( 0 , no inflammatory cells; 1 , minimal inflammation (scattered inflammatory cells close to the junctional epithelium); 2, moderate inflammation (numerous inflammatory cells in the gingival connective tissue); and 3, severe inflammation).

Soft Tissue Soluble Mediator Expression. The right maxilla with both soft tissue and bone were subjected to bead beating at two 2 min minute intervals with 2 min of cooling in between using $1.0 \mathrm{~mm}$ diameter zirconia silica beads (BioSpec, Bartlesville, OK) in cell extraction buffer (ThermoFisher, Waltham, MA) prepared with a protease inhibitor cocktail (mini cOmplete, Roche, Basel, Switzerland) and PMSF protease inhibitor (Abcam, Cambridge, United Kingdom) to allow for dissociation and lysis of all soft tissue while leaving the hard tissues intact. MILLIPLEX® Multiplex Assays (EMD Millipore, Billerica, MA) were used to probe resulting lysates for 
TNFa, IL6, IL8, TGFß1, and IL10, according to the manufacturer protocols. Data was acquired on a Luminex $200 \AA$ system running XPONENT® 3.1 software (Luminex, Austin, TX) and analyzed using a 5-paramater logistic spline-curve fitting method using Milliplex® Analyst V5.1 software (Vigene Tech, Carlisle, MA). Data was acquired on a spectrophotometer and analyzed using liner standard curves. All data are presented as $\mathrm{pg} / \mathrm{ml}$ normalized to total protein as determined by BCA assay (Thermo Scientific Pierce, Waltham, MA).

Real-time PCR of Bacterial Specific 16S Expression. On the first day of infection, prior to infection, and at the beginning of each week of infections, samples of the oral environment were taken with calcium alginate swabs (Fisher Scientific). After which the gDNA was isolated using a DNeasy Kit (Qiagen) according to the manufacturers' instructions. The gDNA was then probed for $P$. gingivalis 16S, A. actinomycetemocomitans $16 S$ and total $16 \mathrm{~S}$ using real time PCR. The percentage of $A$. actinomycetemocomitans $16 \mathrm{~S}$ and $P$. gingivalis $16 \mathrm{~S}$ within the total $16 \mathrm{~S}$ compartment was calculated using the following formula: ct value of total $16 \mathrm{~S} / \mathrm{ct}$ value of $A$. actinomycetemocomitans or $P$. gingivalis 16S. 16s rRNA For: AGA GTT TGA TCC TGG CTC AG Rev: ACG GCT ACC TTG TTA CGA CTT; Pg For: CTT GAC TTC AGT GGC GGC AG; Rev: AGG GAA GAC GGT TTT CAC CA; Aa For: GTT TAG CCC TGG CCG AAG Rev: TGA CGG GCG GTG TGT ACA AGG

Statistical Analysis. Statistical analyses were performed using Prism Software (GraphPad Software, Inc; La Jolla, CA) where one-way ANOVA with Bonferroni's multiple comparisons test was used to determine significance when comparing groups of three or more while Student's $t$ test was used when comparing two groups. $P$ value $<0.05$ was considered significant.

\section{RESULTS}

Loss of MyD88 expression in oral epithelial cells results in exacerbated alveolar bone loss following polymicrobial induction of periodontal disease. The hallmark of periodontal disease is alveolar bone loss, therefore, to evaluate if loss of MyD88 expression within the oral epithelial cells had any effect on periodontal disease, the maxillary bone volume in oral epithelial cell MyD88-sufficient and MyD88-deficient mice following oral polymicrobial infection was evaluated. As expected, compared to uninfected mice (representative data Fig. 1A), polymicrobial infection of B6 mice induced significant bone loss (in Fig. 1C; data summarized in Fig. 11). Similarly, when compared to uninfected counterparts (Fig 1B), polymicrobial infection of $\mathrm{B} 6^{\mathrm{K} 5 \mathrm{Cre} \text { MyD88 }}$ mice with oral epithelial cell sufficient expression of MyD88 also induced significant bone loss (Fig 1D; data summarized in Fig. 1I). Importantly the bone volume observed in uninfected and infected 
nontransgenic B6 mice (Fig. 1A, C), was similar to those levels observed in uninfected and infected $\mathrm{B}^{\mathrm{K} 5 \mathrm{Cre} . \mathrm{MyD} 88}$ mice (Fig. 1B, D; data summarized in Fig. 1I), suggesting that the introduction of transgenes does not affect bone volume.

Conversely, following polymicrobial infection, $\mathrm{B} 6^{\mathrm{K} 5 \mathrm{Cre} \cdot \mathrm{MyD} 88}$ mice lacking oral epithelial cell expression of MyD88 presented with significantly more bone loss (Fig. 1H) than both infected non transgenic B6 mice (Fig $1 \mathrm{G}$ ) and infected B6 ${ }^{\mathrm{K} 5 \mathrm{Cre} \cdot \mathrm{MyD} 88}$ mice with sufficient oral epithelial cell expression of MyD88 (Fig. 1D; data summarized in Fig. 1I). B6 ${ }^{\mathrm{K} 5 \mathrm{Cre} . M y D 88}$ mice lacking oral epithelial cell MyD88 expression also presented with statistically significant bone loss even in the absence of infection (Fig. 1F) when compared to uninfected B6 mice with sufficient oral epithelial cell expression of MyD88 (Fig. 1E, data summarized in Fig. 1I). This observed bone loss was not due to administration of RU486 (i.e. hormone) used to induce the knockdown of MyD88, as uninfected B6 mice treated with RU486 did not demonstrate any bone loss (Fig. 1E). In addition, a difference in bone volume was not observed in infected B6 mice treated with RU486 (Fig. 1G) compared to those infected in the absence of hormone (Fig. 1C, data summarized in Fig. 1I).

Loss of oral epithelial cell MyD88 expression results in increased cellular infiltrate. The initiation and progression of periodontal disease is associated with soft tissue inflammation that results in changes to its architecture, including loss of epithelial attachment and formation of long junctional epithelium as a result of changes in cellular infiltrate (Ebersole et al., 2016). Thus, in order to evaluate if loss of MyD88 expression within the oral epithelial cells had any effect on cellular infiltration, mandibles were histologically evaluated and the level of gross inflammatory infiltrate semi-quantified (Fig. 1J).

Similarly, to that observed with alveolar bone loss, compared to uninfected mice, polymicrobial infection of B6 mice resulted in a significant cellular infiltrate (Fig. 1J). Similarly, when compared to uninfected counterparts, polymicrobial infection of $\mathrm{B}^{\mathrm{K} 5 \mathrm{Cre} \cdot \mathrm{MyD} 88}$ mice with oral epithelial cell sufficient expression of MyD88 also induced a significant infiltrate (Fig. 1J). Again the cellular infiltrate observed in uninfected and infected nontransgenic B6 mice, was similar to that observed in uninfected and infected B6 ${ }^{\mathrm{K} 5 \mathrm{Cre} . \mathrm{MyD} 88}$ mice (Fig. 1J), suggesting that the introduction of transgenes did not affect disease progression.

Following polymicrobial infection, B6 ${ }^{\mathrm{K} 5 \mathrm{Cre} . \mathrm{MyD} 88}$ mice lacking oral epithelial cell expression of MyD88 presented with a significantly higher inflammatory score than both infected non transgenic B6 mice and infected $B 6^{\mathrm{K} 5 \mathrm{Cre} . \mathrm{MyD} 88}$ mice with sufficient oral epithelial cell expression of MyD88 (Fig. 1J). B6 ${ }^{\mathrm{K} 5 \mathrm{Cre} . \mathrm{MyD} 88}$ mice 
lacking oral epithelial cell MyD88 expression also presented with a significant higher inflammatory score even in the absence of infection when compared to uninfected B6 mice with sufficient oral epithelial cell expression of MyD88 (Fig. 1J). Once again, this elevated inflammatory score was not due to administration of RU486, as uninfected B6 mice treated with RU486 did not present with inflammatory infiltration (Fig. 1J). In addition, a difference in inflammatory score was not observed in infected B6 mice treated with RU486 compared to those infected in the absence of hormone (Fig. 1J).

\section{Oral Epithelial Cell MyD88-/- Results in Exacerbated Pro-Inflammatory Cytokine Production.} Compared to uninfected mice, polymicrobial infection of B6 mice resulted in elevated soft tissue expression of TNFa, IL1 $\beta$, and IL6 (Fig. 2A-C) and lower soft tissue expression of IL10 and TGF $\beta$ (Fig. 2D-E). Similarly, when compared to uninfected counterparts, polymicrobial infection of $\mathrm{B} 6^{\mathrm{K} 5 \mathrm{Cr} . \mathrm{MyD} 88}$ mice with oral epithelial cell sufficient expression of MyD88 also induced similar elevations in TNFa, IL1 $\beta$, and IL6 (Fig. 2A-C) and suppression of IL10 and TGF $\beta$ (Fig. 2D-E). Again the changes in soluble mediator expression observed in uninfected and infected nontransgenic B6 mice, was similar to that observed in uninfected and infected $\mathrm{B} 6^{\mathrm{K} 5 \mathrm{Cre} . \mathrm{MyD} 88}$ mice (Fig. 2), suggesting that the introduction of transgenes did not affect disease progression.

Following polymicrobial infection, $\mathrm{B} 6^{\mathrm{K} 5 \mathrm{Cr} e . M y D 88}$ mice lacking oral epithelial cell expression of MyD88 presented with a more robust expression of TNFa, IL1 $\beta$, and IL6 (Fig. 2A-C) when compared to both infected non transgenic $\mathrm{B} 6$ mice and infected $\mathrm{B} 6^{\mathrm{K} 5 \mathrm{Cr} \text {.MyD88 }}$ mice with sufficient oral epithelial cell expression of MyD88 (Fig. 2A-C). This was coupled with a more robust decrease in soft tissue expression of IL10 and TGF $\beta$ (Fig. 2D-E). Again, B6 $6^{\mathrm{K} 5 \mathrm{Cr} . \mathrm{MyD} 88}$ mice lacking oral epithelial cell MyD88 expression also presented with mild but statistically significant elevations in TNFa, IL1 $\beta$, and IL6 (Fig. 2A-C) concomitant with lower IL10 and TGF $\beta$ (Fig. 2D-E) within the soft tissues in the absence of infection when compared to uninfected B6 mice with sufficient oral epithelial cell expression of MyD88 (Fig. 2). Once again, the alterations in the soluble mediator milieu was not due to administration of RU486, as uninfected B6 mice treated with RU486 did not present with these patterns of expression (Fig. 2). In addition, a difference in expression patterns or levels was not observed in infected B6 mice treated with RU486 compared to those infected in the absence of hormone (Fig. 2).

Oral Epithelial Cell MyD88-/- Results in Elevated P. gingivalis and A. actinomycetemocomitans loads. The non-resolving inflammation responsible for periodontal disease progression is induced by bacterial 
insult (Ebersole et al., 2016), therefore, in order to evaluate if loss of MyD88 expression within the oral epithelial cells had any effect on $P$. gingivalis, A. actinomycetemocomitans or total bacterial load (Fig. 3), species specific and total $16 \mathrm{~S}$ levels were evaluated in oral swabs from all experimental groups. As expected, the uninfected groups of mice had no detectable levels of $P$. gingivalis or $A$. actinomycetemocomitans 16 S (Fig. 3). In addition, the total bacterial loads in all experimental groups were not statistically different (data not shown). As observed with all other outcomes the bacterial burden of $P$. gingivalis and $A$. actinomycetemocomitans in infected nontransgenic B6 mice, was similar to that observed in infected $\mathrm{B6}^{\mathrm{K} 5 \mathrm{Cre} . \mathrm{MyD} 88}$ mice (Fig. 3), suggesting that the introduction of transgenes did not affect disease progression. Following polymicrobial infection, B6 ${ }^{\mathrm{K} 5 \mathrm{Cre} . \mathrm{MyD} 88}$ mice lacking oral epithelial cell expression of MyD88 presented with statistically higher loads of $P$. gingivalis and $A$. actinomycetemocomitans infected than non transgenic B6 mice and infected B6 ${ }^{\mathrm{K} 5 \mathrm{Cre} . \mathrm{MyD} 88}$ mice with sufficient oral epithelial cell expression of MyD88 (Fig. 3). Once again, elevations in this bacterial burden was not due to administration of RU486, as infected B6 mice treated with RU486 did not present with elevations in pathogenic load compared to those infected in the absence of hormone (Fig. 3). 


\section{DISCUSSION}

Direct tissue damage by periodontal pathogens and their virulence factors is not the only mechanism of periodontal disease progression, nor is periodontal disease simply an aberrant or idiopathic inflammatory response (Gaffen \& Hajishengallis, 2008; Hajishengallis, 2009). Rather, subgingival bacteria initiate and sustain a non-resolving inflammation that is ineffective at controlling the infection (Gaffen \& Hajishengallis, 2008). There are multiple murine models utilized to study periodontal disease etiology including but not limited to, subgingival LPS injection, third molar ligature (using ligatures soaked in mono and polymicrobial cultures), and oral gavage (again mono and polymicrobial) models (Graves, Kang, Andriankaja, Wada, \& Rossa, 2012). Here we utilized a polymicrobial oral gavage method incorporating the two periodontal pathogens, $P$. gingivalis and A. actinomycetemocomitans, as well as well-characterized strains of these pathogens, which in our hands induce robust alveolar bone loss in mice. Microbes are initially detected by pattern recognition receptors (PRRs), such as TLRs, whereby they detect highly conserved pattern associated molecular patterns (PAMPs). TLRs are expressed on numerous types of cells, but are not restricted to cells of the immune system, and thus expressed on a variety cells within the periodontium (Mahanonda \& Pichyangkul, 2007). Of the cells of the periodontium that express TLRs, the oral epithelium act as the first line of defense against microbial invasion. As TLRs recognize both commensal and pathogenic organisms, their engagement can induce inhibition of the immune system in the presence of commensal microbes as well as upregulate the production of proinflammatory cytokines in the presence of pathogenic conditions. Indeed, it has been demonstrated that epithelial cells actually require TLR stimulation by the commensal organisms for the maintenance of oral health as well as appropriate and timely clearance of infection (Hatakeyama et al., 2003; Neiva, Calderon, Alonso, Panagakos, \& Wallet, 2014; Wallet et al., 2013). Because they are involved in both suppressing and inducing the immunologic response to microbial detection, TLRs of the epithelial cells play an important role in maintaining the balance of homeostasis and inflammation.

Indeed, data presented here demonstrate that MyD88-dependent innate sensing by oral epithelial cells is key and not only important in controlling oral inflammation, but maintaining immunological homeostasis within the soft and hard tissues of the oral cavity. Specifically, data presented here demonstrated that loss of oral epithelial cell MyD88-expression resulted in exacerbated expression of pro-inflammatory cytokines such as IL6, IL1ß and TNFa. IL6 has significant pleiotropic activities including induction of 1) enzymes from 
granules, 2) the metabolism of reactive oxygen species (ROS), and the 3) chemotaxis and retainment of immune cells, all of which can amplify the inflammatory cascade (Rosenkilde \& Schwartz, 2004). Importantly, IL6, IL1 $\beta$ and TNFa can also induce osteoclastogenesis and osteoclast activation (Bendre et al., 2003). On the other hand, additional data presented here demonstrated that loss of oral epithelial cell MyD88-dependent results in a loss of TGF $\beta 1$ and IL10 expression under conditions of infection as well as homeostasis. TGF $\beta$ and IL10 cooperatively suppress innate immune cell activation and the induction and expansion of adaptive immunity (Ebersole et al., 2016). Specifically, this milieu suppresses the production of IL6, IL1 $\beta$, and TNF a and expression of major histocompatibility complex II (MHCII), thus indirectly affecting the activation of adaptive immunity (Ebersole et al., 2016). This milieu can also directly affect adaptive immunity by inhibiting the proliferation and expansion of most adaptive immune cells (Letterio \& Roberts, 1998). Additionally, TGF $\beta$ can also induce the synthesis of bone matrix proteins by osteoblasts as well as most major extracellular matrix proteins necessary for the wound healing. Thus, together these data demonstrate that loss of this homeostatic cytokine milieu coupled with the exacerbation of a pro-inflammatory milieu drives both soft and hard tissue damage while also preventing timely and proper wound healing (Letterio \& Roberts, 1998), contributing, at least in part, to the exacerbation of soft and hard tissue damage observed as well.

Oral epithelial cells directly and indirectly contribute to the control of commensal flora as well as plaque accumulation through the secretion of anti-microbial peptides and chemokines respectively where by activation of TLRs by commensal bacteria is critical for this maintenance of homeostasis (Gorr, 2012). Indeed, our data demonstrates that MyD88-expression of oral epithelial cells is key in controlling at the pathogenic bacterial load. While not investigated in this study, this could be in part be through decreased MyD88-dependent induction of antibacterial $\beta$-defensins, cathelicidin and calprotectin as well as the neutrophil chemoattractant IL8 (Gorr, 2012). In health, gingival tissues contain low numbers of innate and adaptive immune cells recruited to the periodontal pocket where they assist in maintaining homeostasis between the host periodontal tissues and the bacterial plaque (Ebersole et al., 2013).

Oral epithelial cells also manage the balance among tolerance, protective immunity, and inflammation through directing immune cell phenotype and function. While the data presented here demonstrate that the nature of oral epithelial cell response can dictate the soluble mediator environment, its direct and indirect effects on individual innate and adaptive immune cell populations was not elucidated. In early PD lesions, 
there is an acute neutrophil response and a slight increase in lymphocytic cells. In chronic disease, there is an increase in antigen presenting cells (APCs), including macrophages (MØ) (Lappin, MacLeod, Kerr, Mitchell, \& Kinane, 2001; Navarrete et al., 2014; Okada \& Murakami, 1998). Similarly, the T cell infiltrate increases in which the cytokine profiles are skewed compared to T cells from healthy or gingivitis patients (Ebersole et al., 2013). This again suggests altered immune-regulation or plasticity within the innate and adaptive immune cell compartments. How these three key cells, oral epithelial cells, macrophages and $\mathrm{T}$ cells interact to promote immune plasticity responsible for appropriate (pathogen clearing) and inappropriate (tissue damaging) innate and adaptive immune responses is the future focus of our program. 


\section{ACKNOWLEDGMENTS}

The authors thank the National Institutes of Health/ National Institute of Dental and Craniofacial Research for the financial support (NIH/NIDCR R01DE023567-01 (Wallet); NIH/NIDCR T90 DE021990-02 (Burne)). The authors have no conflicts of interest to report. 


\section{FIGURE LEGENDS}

Figure 1. Loss of MyD88 expression in oral epithelial cells results in exacerbated alveolar bone loss and immune infiltration following polymicrobial induction of periodontal disease. (A-H) Maxillae were imaged under 2X magnification perpendicular to the alveolar bone. Scale bar was calibrated using a predetermined measure at $2 \mathrm{X}$ magnification. The distance between the CEJs of murine posterior teeth and alveolar crest were outlined and measured. Right and left maxillary bone loss was measured on both the lingual and buccal surfaces in mice from all experimental groups. (A) uninfected B6 (MyD88 sufficient) (B) uninfected B6 ${ }^{\text {K5Cre.MyD88 }}$ (MyD88 sufficient) $(C)$ infected B6 (MyD88 sufficient) (D) infected B6 ${ }^{\text {K5Cre.MyD88 }}$ (MyD88 sufficient) (E) uninfected B6 treated with RU486 (MyD88 sufficient) (F) uninfected B6 ${ }^{\mathrm{K} 5 \mathrm{Cre} . M y D 88}$ treated with RU486 (MyD88 deficient) $(G)$ infected B6 treated with RU486 (MyD88 sufficient) $(F)$ infected B6 ${ }^{\mathrm{K} 5 \mathrm{Cre} . \mathrm{MyD} 88}$ treated with RU486 (MyD88 deficient). (I) Data are presented as \% of uninfected B6 bone volume. (J) Quantification of immune cell infiltrate. * $p$ value $<0.05$ vs. uninfected within the same strain; ${ }^{\wedge} p$ value $<0.05$ B6 vs $\mathrm{B}^{\mathrm{K} 5 \mathrm{Cre} \cdot \mathrm{MyD} 88}$. ${ }^{\#} \mathrm{p}$ value $<0.05$ uninfected $\mathrm{B} 6^{\mathrm{K} 5 \mathrm{Cre} \cdot \mathrm{MyD} 88}$ vs. infected $\mathrm{B} 6^{\mathrm{K} 5 \mathrm{Cre} \cdot \mathrm{MyD} 88}$. One Way ANOVA with Bonferoni's multiple comparisons ( $n=7$ /group).

Figure 2. Oral Epithelial Cell MyD88-/- Results in Exacerbated Pro-Inflammatory Cytokine Production. Mandibles were subjected to bead beating to homogenize the soft tissue into a buffer containing protease inhibitors. After which multiplex analysis was used to determine the concentration of indicated cytokines. Concentrations were normalized to total protein. ${ }^{*} p$ value $<0.05$ vs. uninfected within the same strain; ${ }^{\wedge} p$ value $<0.05$ B6 vs B6 ${ }^{\mathrm{K} 5 \mathrm{Cre} . \mathrm{MyD} 88}$. ${ }^{\#} \mathrm{p}$ value $<0.05$ uninfected $\mathrm{B} 6^{\mathrm{K} 5 \mathrm{Cre} . \mathrm{MyD} 88}$ vs. infected $\mathrm{B} 6^{\mathrm{K} 5 \mathrm{Cre} . \mathrm{MyD} 88}$. One Way ANOVA with Bonferoni's multiple comparisons ( $n=7 /$ group)

Figure 3. Oral Epithelial Cell MyD88-/- Results in Elevated $\boldsymbol{P}$. gingivalis and $\boldsymbol{A}$. actinomycetemocomitans bacterial load. Samples of the oral environment were taken with calcium alginate swabs were taken on the last day of the experimental protocol. The percentage of $A$. actinomycetemocomitans 16S and $P$. gingivalis $16 \mathrm{~S}$ within the total $16 \mathrm{~S}$ compartment was calculated using the following formula: ct value of total $16 \mathrm{~S} / \mathrm{ct}$ value of $A$. actinomycetemocomitans or $P$. gingivalis $16 \mathrm{~S}$. ${ }^{*} \mathrm{p}$ value $<0.05$ vs. uninfected 
within the same strain; ${ }^{\wedge} p$ value $<0.05$ B6 vs B6 ${ }^{\text {K5Cre.MyD88 }}$. One Way ANOVA with Bonferoni's multiple comparisons $(n=7 /$ group$)$. 


\section{REFERRENCES}

Bendre, M. S., Montague, D. C., Peery, T., Akel, N. S., Gaddy, D., \& Suva, L. J. (2003). Interleukin-8 stimulation of osteoclastogenesis and bone resorption is a mechanism for the increased osteolysis of metastatic bone disease. Bone, 33(1), 28-37.

Blinkhorn, A., Bartold, P. M., Cullinan, M. P., Madden, T. E., Marshall, R. I., Raphael, S. L., \& Seymour, G. J. (2009). Is there a role for triclosan/copolymer toothpaste in the management of periodontal disease? Br Dent J, 207(3), 117-125.

Dale, B. A., Kimball, J. R., Krisanaprakornkit, S., Roberts, F., Robinovitch, M., O'Neal, R., ... Weinberg, A. (2001). Localized antimicrobial peptide expression in human gingiva. J Periodontal Res, 36(5), 285-294.

Diamond, D. L., Kimball, J. R., Krisanaprakornkit, S., Ganz, T., \& Dale, B. A. (2001). Detection of betadefensins secreted by human oral epithelial cells. J Immunol Methods, 256(1-2), 65-76.

Ebersole, J. L., Dawson, D. R., 3rd, Morford, L. A., Peyyala, R., Miller, C. S., \& Gonzalez, O. A. (2013). Periodontal disease immunology: 'double indemnity' in protecting the host. Periodontol 2000, 62(1), 163-202. doi:10.1111/prd.12005

Ebersole, J. L., Graves, C. L., Gonzalez, O. A., Dawson, D., 3rd, Morford, L. A., Huja, P. E., ... Wallet, S. M. (2016). Aging, inflammation, immunity and periodontal disease. Periodontol 2000, 72(1), 54-75. doi:10.1111/prd.12135

Ford, P. J., Gamonal, J., \& Seymour, G. J. (2010). Immunological differences and similarities between chronic periodontitis and aggressive periodontitis. Periodontol 2000, 53, 111-123.

Gaffen, S. L., \& Hajishengallis, G. (2008). A new inflammatory cytokine on the block: re-thinking periodontal disease and the Th1/Th2 paradigm in the context of Th17 cells and IL-17. J Dent Res, 87(9), 817-828.

Gorr, S. U. (2012). Antimicrobial peptides in periodontal innate defense. Front Oral Biol, 15, 84-98. doi:10.1159/000329673

Graves, D. T., Kang, J., Andriankaja, O., Wada, K., \& Rossa, C., Jr. (2012). Animal models to study hostbacteria interactions involved in periodontitis. Front Oral Biol, 15, 117-132. doi:10.1159/000329675

Hajishengallis, G. (2009). Toll gates to periodontal host modulation and vaccine therapy. Periodontol 2000, 51, 181-207. doi:10.1111/j.1600-0757.2009.00304.x

Hatakeyama, J., Tamai, R., Sugiyama, A., Akashi, S., Sugawara, S., \& Takada, H. (2003). Contrasting responses of human gingival and periodontal ligament fibroblasts to bacterial cell-surface components through the CD14/Toll-like receptor system. Oral Microbiol Immunol, 18(1), 14-23.

Kollisch, G., Kalali, B. N., Voelcker, V., Wallich, R., Behrendt, H., Ring, J., . . Ollert, M. (2005). Various members of the Toll-like receptor family contribute to the innate immune response of human epidermal keratinocytes. Immunology, 114(4), 531-541.

Lappin, D. F., MacLeod, C. P., Kerr, A., Mitchell, T., \& Kinane, D. F. (2001). Anti-inflammatory cytokine IL-10 and T cell cytokine profile in periodontitis granulation tissue. Clin Exp Immunol, 123(2), 294-300.

Letterio, J. J., \& Roberts, A. B. (1998). Regulation of immune responses by TGF-beta. Annu Rev Immunol, 16, 137-161. doi:10.1146/annurev.immunol.16.1.137

Lu, Y. C., Yeh, W. C., \& Ohashi, P. S. (2008). LPS/TLR4 signal transduction pathway. Cytokine, 42(2), 145151.

Mahanonda, R., \& Pichyangkul, S. (2007). Toll-like receptors and their role in periodontal health and disease. Periodontol 2000, 43, 41-55. doi:10.1111/j.1600-0757.2006.00179.x

Malkoski, S. P., Cleaver, T. G., Lu, S. L., Lighthall, J. G., \& Wang, X. J. (2010). Keratin promoter based gene manipulation in the murine conducting airway. Int J Biol Sci, 6(1), 68-79.

McCormick, T. S., \& Weinberg, A. Epithelial cell-derived antimicrobial peptides are multifunctional agents that bridge innate and adaptive immunity. Periodontol 2000, 54(1), 195-206.

Navarrete, M., Garcia, J., Dutzan, N., Henriquez, L., Puente, J., Carvajal, P., . . Gamonal, J. (2014). Interferongamma, interleukins- 6 and -4 , and factor XIII-A as indirect markers of the classical and alternative 
macrophage activation pathways in chronic periodontitis. J Periodontol, 85(5), 751-760. doi:10.1902/jop.2013.130078

Neiva, K. G., Calderon, N. L., Alonso, T. R., Panagakos, F., \& Wallet, S. M. (2014). Type 1 diabetes-associated TLR responsiveness of oral epithelial cells. J Dent Res, 93(2), 169-174. doi:10.1177/0022034513516345

Okada, H., \& Murakami, S. (1998). Cytokine expression in periodontal health and disease. Crit Rev Oral Biol Med, 9(3), 248-266.

Page, R. C., \& Kornman, K. S. (1997). The pathogenesis of human periodontitis: an introduction. Periodontol 2000, 14, 9-11.

Rosenkilde, M. M., \& Schwartz, T. W. (2004). The chemokine system -- a major regulator of angiogenesis in health and disease. APMIS, 112(7-8), 481-495. doi:10.1111/j.1600-0463.2004.apm112070808.x

Shibolet, O., \& Podolsky, D. K. (2007). TLRs in the Gut. IV. Negative regulation of Toll-like receptors and intestinal homeostasis: addition by subtraction. Am J Physiol Gastrointest Liver Physiol, 292(6), G1469-1473.

Sugawara, Y., Uehara, A., Fujimoto, Y., Kusumoto, S., Fukase, K., Shibata, K., ... Takada, H. (2006). Toll-like receptors, NOD1, and NOD2 in oral epithelial cells. J Dent Res, 85(6), 524-529.

Wallet, M. A., Calderon, N., Alonso, T. R., Choe, C. S., Catalfamo, D., Lalane, C. J., . . Wallet, S. M. (2013). Triclosan alters antimicrobial and inflammatory responses of epithelial cells. Oral Dis, 19(3), 296302. doi:10.1111/odi.12001

Weinberg, A., Krisanaprakornkit, S., \& Dale, B. A. (1998). Epithelial antimicrobial peptides: review and significance for oral applications. Crit Rev Oral Biol Med, 9(4), 399-414. 
B6
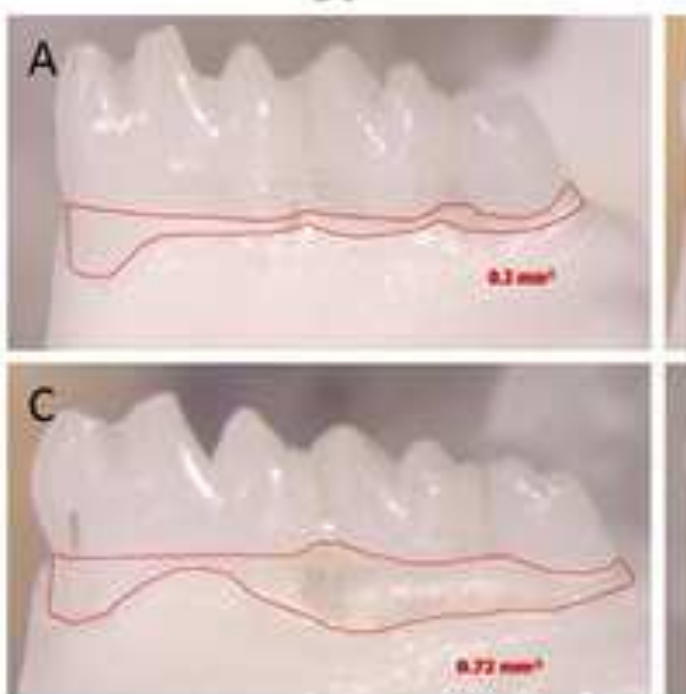

$$
\text { E }
$$$$
\text { ea }
$$

G

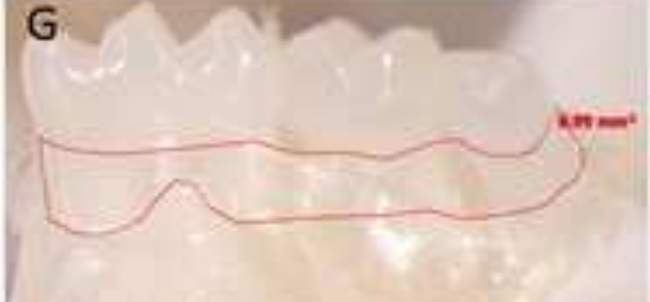

B6 $6^{\text {KSCre.MyDs8plox }}$
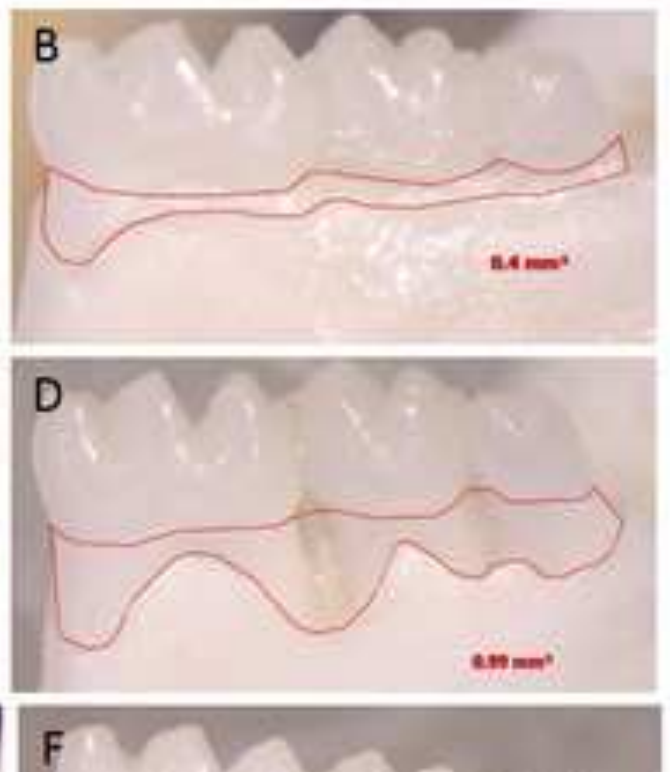

ax

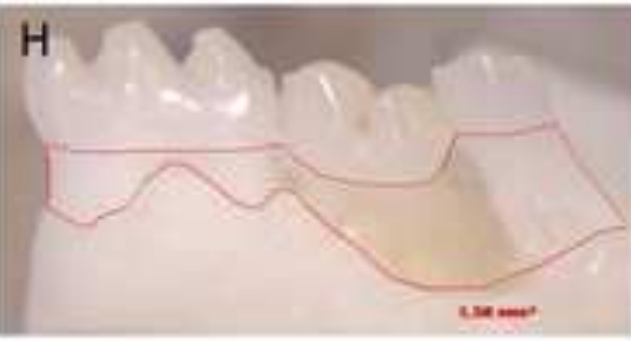

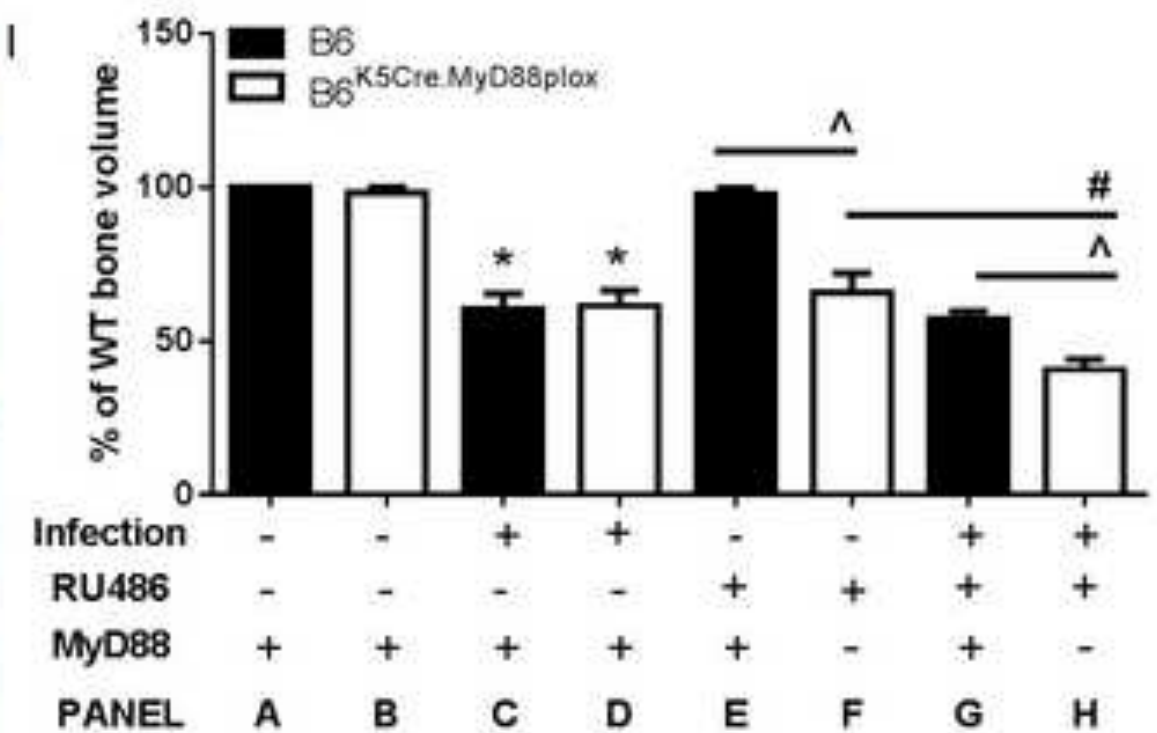

PANEL A B C D E $F$ G

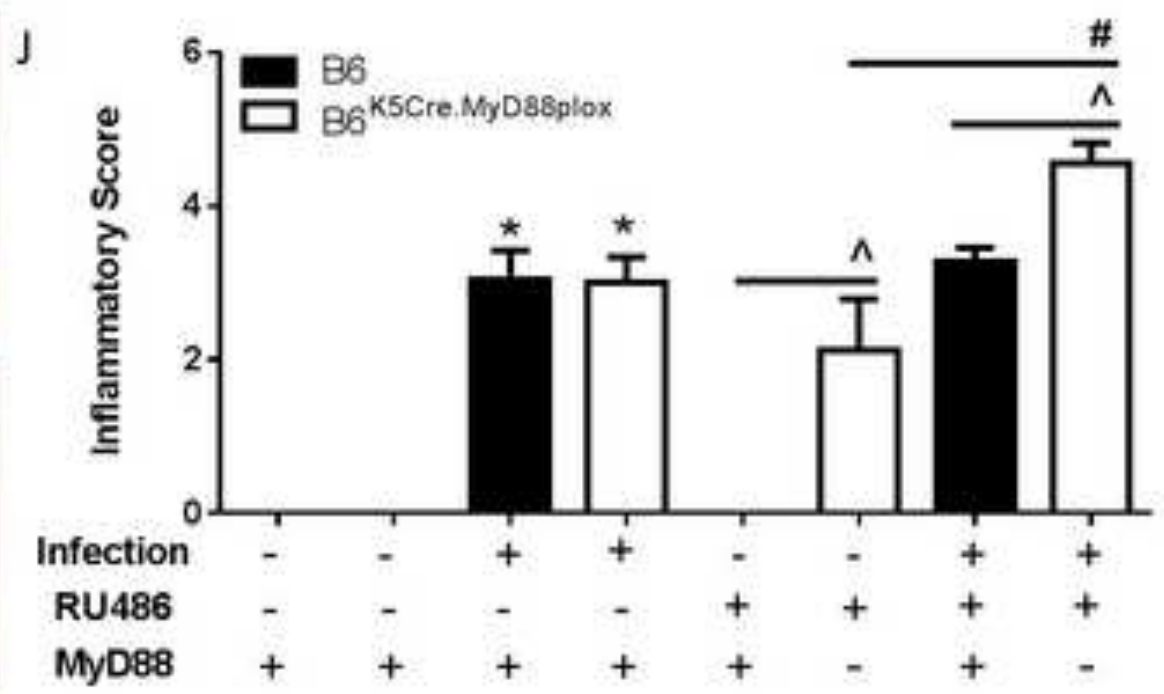



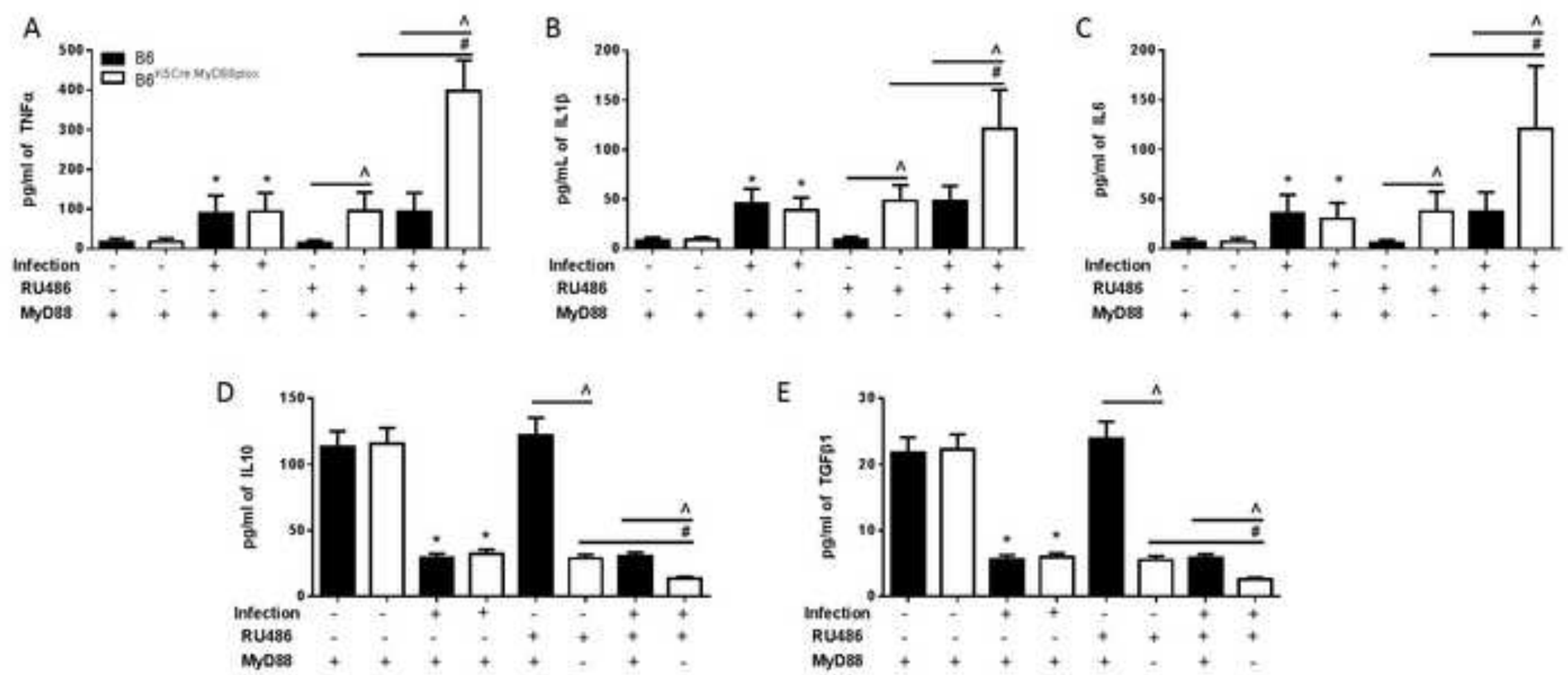

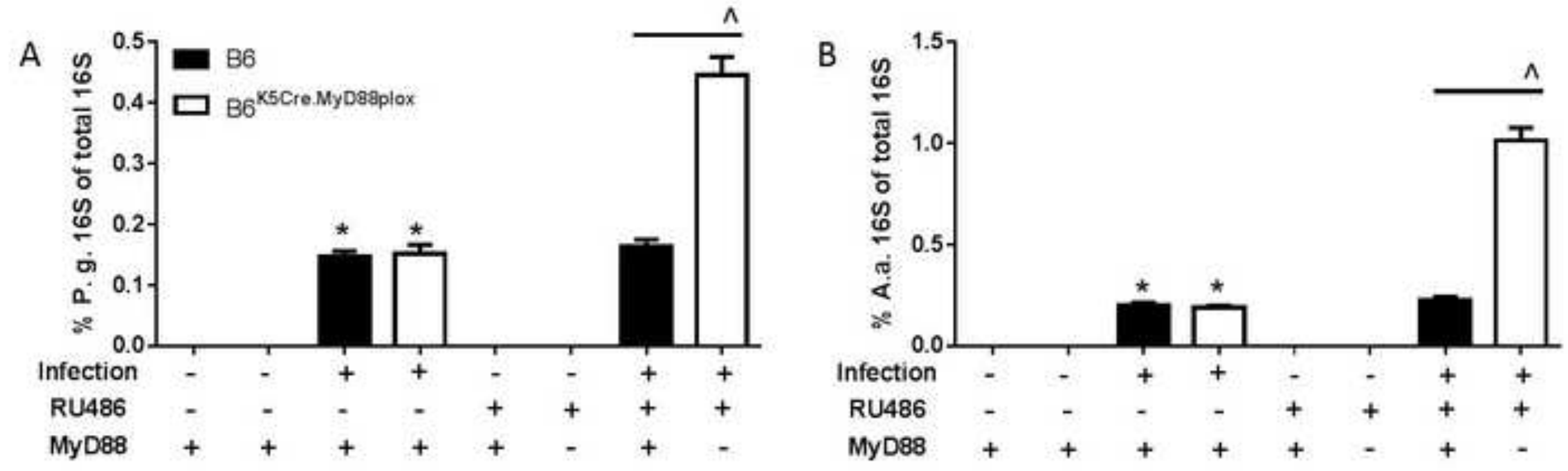